\title{
Research on the method of MPPT of photovoltaic systems based on PWM control
}

\author{
Feng Yingbei ${ }^{1, \text { a }}$, Zhang Jian ${ }^{2, b}$, Lv Nawei ${ }^{3, c}$ \\ ${ }^{1}$ School of Electrical Engineering, Zhengzhou University, Zhengzhou 450001, China. \\ afengyingbei@163.com, 'bhangjian63@zzu.edu.cn, 'Ivnawei@stu.zzu.edu.cn
}

\begin{abstract}
Keywords: PV, maximum power point, PWM control, Newton interpolation.
Abstract. In order to make PV systems more efficient and maximize the output power, this paper proposed a new MPPT algorithm for photovoltaic power generation, which combined PWM control and Newton quadratic interpolation, based on the analysis of the traditional maximum power point tracking (MPPT) control algorithm. The whole system was modeled and simulated on MATLAB/Simulink, and the results validate that the proposed method can search the maximum power point fast and avoid the oscillation around the maximum power point. The model used load which could be changed with PWM instead of boost circuit to avoid the interference of too much power electronics.
\end{abstract}

\section{Introduction}

With the increasing of global pollution, the gradual enhancement of human environmental awareness and the acute shortage of traditional energy sources such as coal and oil, new renewable clean energy sources such as solar energy, wind energy and water energy are increasingly favored by people.Solar PV cells are a kind of semiconductor devices that convert solar energy directly into electrical energy ${ }^{[1]}$. However, the output power of PV modules varies with the irradiance of the sun and the temperature of the solar panels. In order to maximize the output power of the PV system, it is necessary to track the maximum power point of the PV array. The analysis shows that for a constant load, photovoltaic cells have the only operating point to generate the maximum power, the point known as the MPP point. Therefore, a MPPT is an essential part of PV system to ensure that inverters operate at the maximum power of the PV array ${ }^{[2]}$.

At present, a variety of algorithms can be used to determine the maximum power point of the system $^{[3-5]}$, most of these methods from the control voltage analysis, but its regulation process can not directly act on the voltage, there are certain flaws. Based on the principle of MPPT control algorithm, an improved perturbation observation algorithm is proposed. Based on the relationship between power and duty cycle (PWM), the resistance can be intuitively adjusted by controlling the duty cycle and adjusting the domain. Near the power point, and then combined with Newton interpolation ${ }^{[6-7]}$, accurate positioning to the maximum power point, effectively avoid the shock. Finally, the correctness and superiority of the algorithm are proved by simulation.

\section{PV model}

The analysis of this paper is about the external characteristics of photovoltaic cells, using the literature [1] to improve the behavior model, the output characteristics is expressed as:

$$
\begin{aligned}
& I=I_{s c}^{\prime}\left[1-C_{1}\left(e^{V /\left(C_{2} V_{o c}^{\prime}\right)}-1\right)\right] \\
& C_{1}=\left(1-I_{m}^{\prime} / I_{s c}^{\prime}\right) e^{-V_{m}^{\prime} /\left(C_{2} V_{o c}^{\prime}\right)} \\
& C_{2}=\left(V_{m}^{\prime} / V_{o c}^{\prime}\right)\left[\ln \left(1-I_{m}^{\prime} / I_{s c}^{\prime}\right)\right]^{-1} \\
& \left\{\begin{array}{l}
I_{s c}^{\prime}=I_{s c} \Delta I \\
I_{m}^{\prime}=I_{m} \Delta I
\end{array}\right.
\end{aligned}
$$




$$
\begin{gathered}
\left\{\begin{array}{l}
V_{o c}^{\prime}=U_{o c} \Delta V \\
V_{m}^{\prime}=U_{m} \Delta V
\end{array}\right. \\
\Delta I=\frac{S}{S_{\text {ref }}}\left[1+a\left(T-T_{r e f}\right)\right] \\
\Delta V=\left[1-c\left(T-T_{\text {ref }}\right)\right] \ln \left[e+b\left(S-S_{\text {ref }}\right)\right]
\end{gathered}
$$

Where:

V Cell Output Voltage

I Cell Output Current;

$C_{1}, C_{2} \quad$ Correction factor

$a, b, c \quad$ Compensation factor, and $\mathrm{a}=0.0025, \mathrm{~b}=0.0005, \mathrm{c}=0.00288$ in this paper.

The simulation model is presented in Fig1 in Matlab / Simulink.

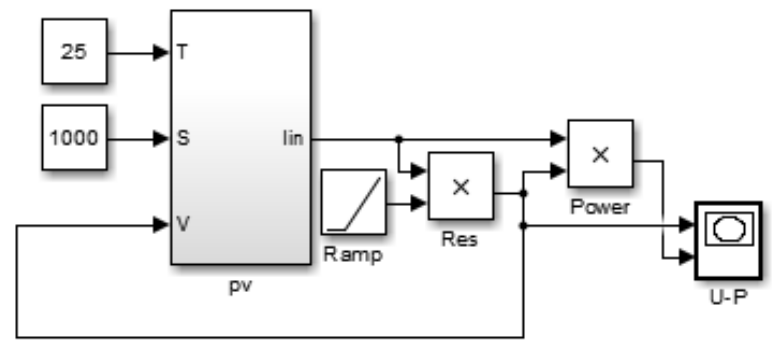

Fig.1 Photovoltaic cell model

Based on standard test conditions (STC) at $1000 \mathrm{~W} / \mathrm{m}^{2}$ irradiance and $25^{\circ} \mathrm{C}$ of surface temperature, the output characteristics is presented in Fig2 and Fig3:

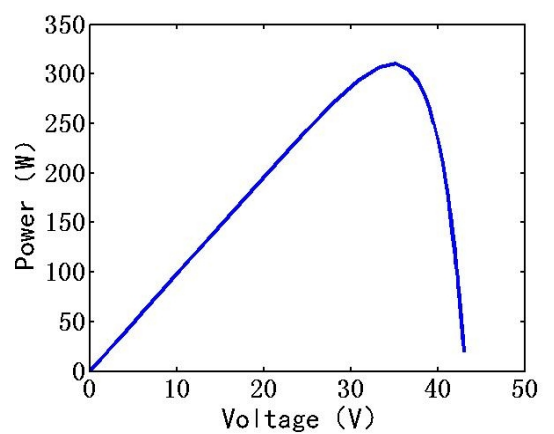

Fig.2 P-V curve of Photovoltaic cell

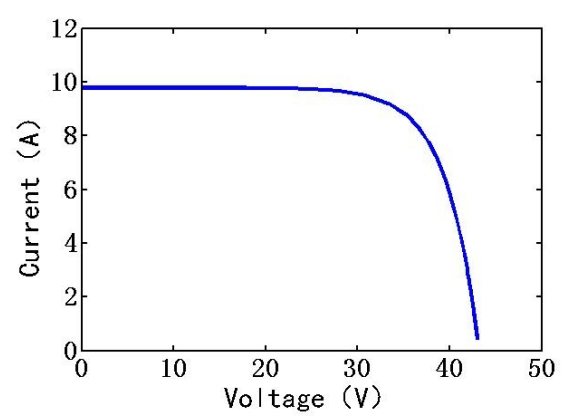

Fig.3 I-V curve of Photovoltaic cell

PV arrays exhibits non-linear varying, and the output power $\mathrm{P}$ has a unique maximum value under certain operating conditions, when the external environment is constant,. The purpose of the MPPT is to find the maximum power point and keep the photovoltaic cell working at that point.

\section{PV MPPT algorithm}

PWM control. By controlling the load impedance of photovoltaic cells to achieve the maximum power point tracking when the external environment can not control is the essence of MPPT algorithm, The control strategy is to increase or decrease the duty cycle to control the load which can influence voltage and then change the value of power, according to the comparing results of the values of power twice in succession which are calculated from the output voltage and current of photovoltaic cells. Therefore, the tracking process will be more directly by analyzing the relationship between PWM and power. Figure 4 shows the change of the power when the duty cycle changes from 0 to 1 in the model used in this paper. It can be seen that the maximum power points are the same point in Fig. 2 and Fig. 4. 
Newton interpolation. It is difficult to derive the function of $\mathrm{P}$ to PWM on $[0,1]$ from Fig.4, and interpolation is one of the important methods of curve fitting. So a simple fitting curve of the function can be obtained by using Newton interpolation to get the maximum power point quickly based on the sampled data.

The value of PWM is denoted by D. From figure 4, we can see that $\mathrm{dp} / \mathrm{dD}=0$ at MPP, so we can get a power $\mathrm{P}$ by three sampling points with quadratic Newton interpolation:

$$
P=a D^{2}+b D+c
$$

According to $\mathrm{d} p / \mathrm{d} D=2 \mathrm{a} D+\mathrm{b}=0$, the value of PWM at MPP is out, then the maximum power can be determined.

\section{Improving MPPT algorithm}

By using the idea of quadratic Newton interpolation, the maximum power point can be determined in one step by finding three points near the MPP. At the same time, the tracking speed can be greatly improved, and the oscillation near the MPP can be avoided to the utmost extent in this way. However, the Sampling points (Dt-2, Pt-2), (Dt-1, Pt-1), (Dt, Pt) must cover both sides of the MPP to avoid the error of curve fitting as shown in Fig.5。

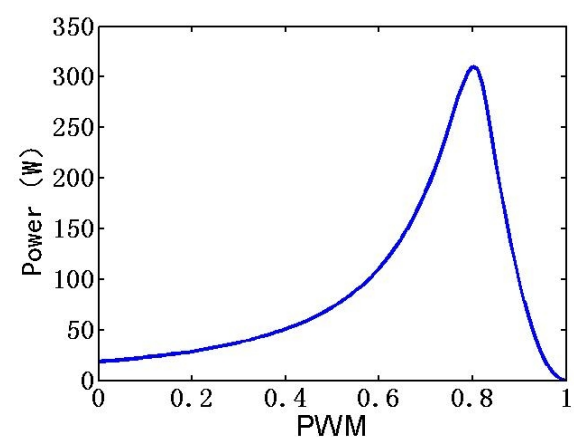

Fig.4 The curve of PWM-P

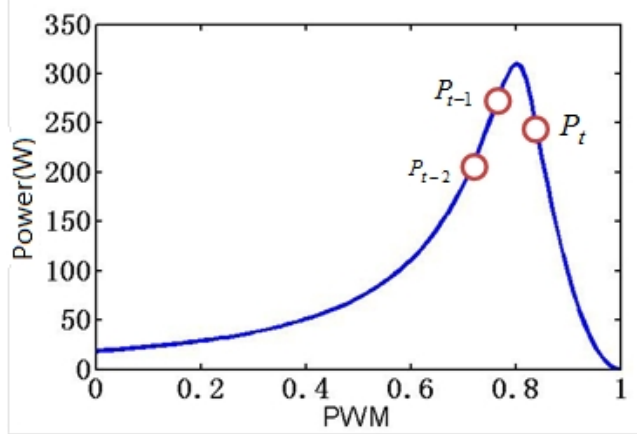

Fig.5 The position of effective sampling point

The difference quotients in quadratic Newton interpolation are expressed as:

$$
\begin{aligned}
& P\left[D_{t-2}, D_{t-1}\right]=\frac{P_{t-1}-P_{t-2}}{D_{t-1}-D_{t-2}} \\
& P\left[D_{t-1}, D_{t}\right]=\frac{P_{t}-P_{t-1}}{D_{t}-D_{t-1}} \\
& P\left[D_{t-2}, D_{t-1}, D_{t}\right]=\frac{P\left[D_{t-1}, D_{t}\right]-P\left[D_{t-2}, D_{t-1}\right]}{D_{t}-D_{t-2}}
\end{aligned}
$$

The quadratic interpolation polynomial is expressed as:

$$
N_{2}(D)=P_{t-2}+P\left[D_{t-2}, D_{t-1}\right)\left(D-D_{t-2}\right)+P\left[D_{t-2}, D_{t-1}, D_{t}\right]\left(D-D_{t-2}\right)\left(D-D_{t-1}\right)
$$

According to $d p / d(D)=0$ at MPP , the PWM value at MPP is expressed as:

$$
D_{m}=\frac{1}{2}\left[\left(D_{t-2}+D_{t-1}\right)-\frac{P\left[D_{t-2}, D_{t-1}\right]}{P\left[D_{t-2}, D_{t-1}, D_{t}\right]}\right]=\frac{1}{2}\left(D_{t-2}+D_{t-1}\right)-\frac{\left(P_{t-1}-P_{t-2}\right)\left(D_{t-1}-D_{t-2}\right)}{P_{t}-2 P_{t-1}+P_{t-2}}
$$


Based on the combination of PWM control and Newton interpolation, this paper puts forward a new improved algorithm. First of all find three points whose values of power are calculated in the process of PWM growing from 0 to 1 step by step and which must meet the requirments shown in fig.5. The MPP is bound to be in the range of three points contains. Then we can calculate the value of power of the MPP by quadratic Newton interpolation. The flowchart of the modified MPPT algorithm is shown in Fig. 6:

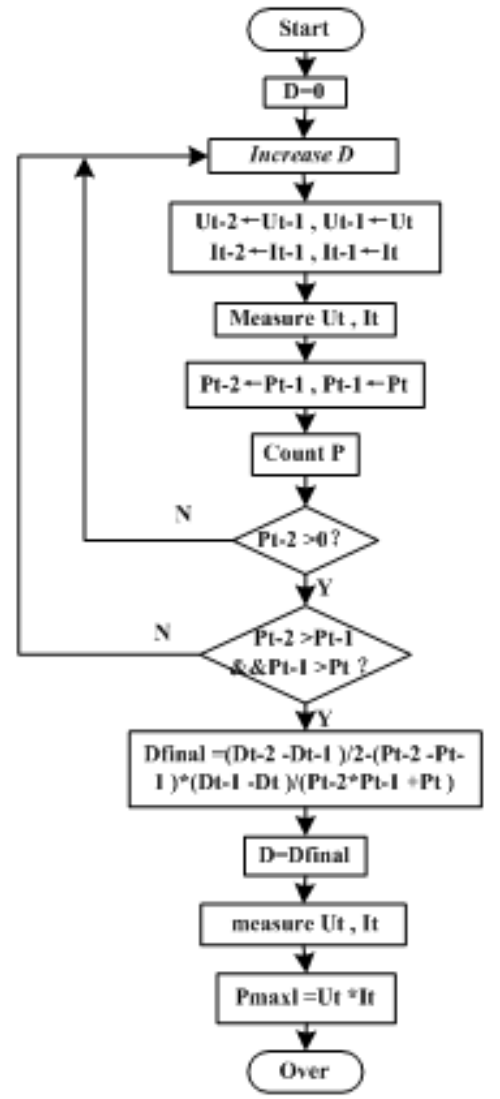

Fig.6 Flowchart of improved algorithm

\section{Simulation and analysis}

To verify the feasibility and correctness of the improved algorithm , a simulation model is built in Matlab/Simulink according to the mathematical model of PV cell, which has the following manufacturing specification: $\mathrm{Voc}=43.2 \mathrm{~V}, \mathrm{Isc}=9.8 \mathrm{~A}$, voltage at maximum power $=34.4 \mathrm{~V}$, current at maximum power $=9.02 \mathrm{~A}$. The simulated $\mathrm{I}-\mathrm{V}$ characteristic and the MPP, for a $1000 \mathrm{~W} / \mathrm{m}^{2}$ radiation and 25 of surface temperature, are presented in the Fig.7. The model uses load which can be change with PWM instead of boost circuit to avoid the interference of too much power electronics. The results are shown in Fig.8.

Figure 8 shows that the system can keep working at the point and there is no oscillation when the maximum power point is reached in 0.08 seconds.

\section{Conclusions}

In this paper, Based on the analysis of the traditional MPPT control algorithm, the relationship between the PWM output power and the output power of the system is discussed in detail. And this paper puts forward a new improved MPPT algorithm for photovoltaic cells which is based on the PWM control and the quadratic Newton interpolation.

(1)In this algorithm, three effective points near the maximum power point are found by the PWM perturbation, and the quadratic fitting function of the power is obtained by Newton interpolation method, and the maximum output power of the photovoltaic power generation system is obtained. 
(2)The whole control process is simple, clear and intuitive. It can quickly find the maximum power point and avoid the oscillation near the maximum power point.

(3)The correctness and superiority of the algorithm are verified by the introduction of simulation.

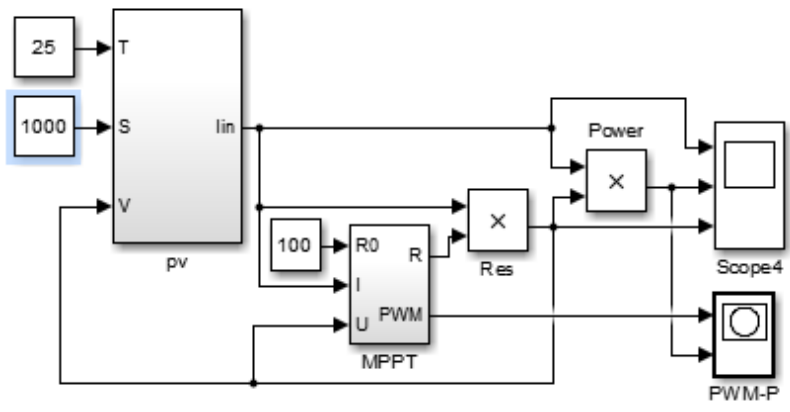

Fig.7 PV MPPT algorithm simulation on Matlab/Simulink

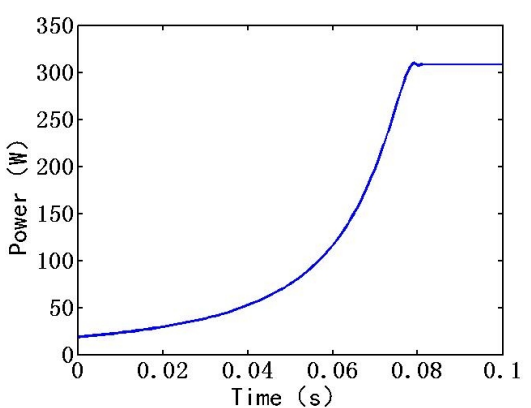

(a)P-t

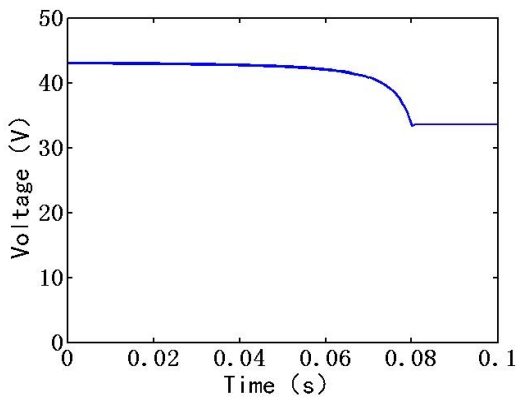

(b) $\mathrm{V}-\mathrm{t}$

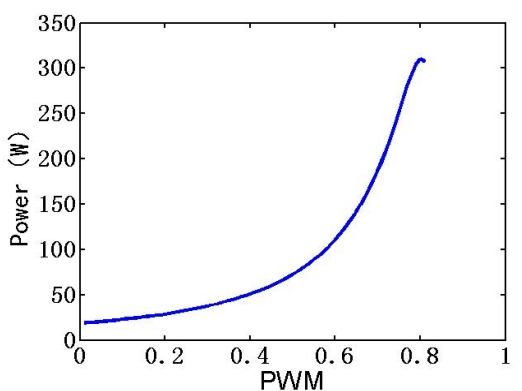

(c)P-PWM

Fig.8 Simulation results of the improved algorithm

\section{References}

[1] Jiao Yang and Song Qiang: Power System Technology, 2010, 11:p.198-202. In Chinese.

[2] Han Tao. Simulation research of Photovoltaic Power generation system based on Matlab[D]. Shandong University of Technology, 2014. In Chinese.

[3] Ahmad Al-Diab and Constantinos Sourkounis: 2010, 12th International Conference on Optimization of Electrical and Electronic Equipment, OPTIM 2010: p.1097-1102.

[4] Hang Haifeng, Yang Wei and Zhu Wenyan: Power System Protection and Control, 2014, 09:p.110-114. In Chinese.

[5] Zhang Lin, Guo Qiangang and Zhou Xifeng: Electrical Measurement \& Instrumentation, 2014, 02:p.27-31. In Chinese.

[6] Gao Song, Ma Hongli, He Ning and Chen Chaobo: Control Engineering of China, 2015, 08:p.120-124. In Chinese.

[7] Wu Guohua and Li Xiangdong: Power Technology, 2015, 07:p.1432-1434+1437. In Chinese. 